\title{
Reduction for Ordinary and Partial Differential Equations by Using Lie Group
}

\author{
Eman Ali Hussain ${ }^{a} \quad$ Zainab Mohammed Alwan $^{\mathrm{b}}$ \\ ${ }^{a}$ Department of Mathematics, College of Science, University of AL-Mustansiriyah, Iraq \\ ${ }^{b}$ Department of Mathematics, College of Science, University of AL-Mustansiriyah, Iraq \\ dreman@uomustansiriyah.edu.iq lionwight_2009@uomustansiriyah.edu.iq
}

Submission date:- 13/12/2018 Acceptance date:- 15/1/2019 Publication date:- 3/4/2019

Keywords: Lie group, invariance, PDEs, prolongation, reduction, ODEs.

\begin{abstract}
In this publication, We have done Lie group theory is applied to reduce the order of ordinary differential equations (ODEs) with 1-parameter and reduce a PDEs to ODEs . Also set up algorithm to solve ODEs and PDEs to obtain the exact solution .

\section{Introduction}

The Lie symmetries and their various generalizations have become an inseparable part of the modern physical description of wide range of phenomena of nature from quantum physics to hydrodynamics. Such success of a purely mathematical theory of continuous groups developed by Lie and Engel in 19th century[8] is explained by the remarkable fact that the overwhelming majority of mathematical models of physical, chemical, and biological processes possess nontrivial Lie symmetry. Symmetries can be applied to reduce the order of an ODE . [9] proposed a generalization of Lie's method called the non-classical method of group-invariant solutions. It is well known that the classical Lie symmetry method of point transformations is often used for reducing the number of independent variables in partial differential equation to obtain ordinary one can obtain partial solutions of the equation under study [1], [2], [3]. For partial differential equations, Lie symmetries are used to reduce the equation to an ODE via appropriate reduction variables. These can lead to the group-invariant solutions [4] of PDEs which are so important today[5],[6].
\end{abstract}

\section{Reduction of Order for ODE,[1]}

Consider the $\mathrm{n}^{\text {th }}$ order ODE as:

$G\left(x, y, y^{\prime}, y^{\prime \prime}, \ldots, y^{(n)}\right)=y^{(n)}-g\left(x, y, y^{\prime}, y^{\prime \prime}, \ldots, y^{(n-1)}\right)=0$

Admits the group as

$x^{*}=X(x, y, \in)=x+\in \zeta(x, y)+O\left(\in^{2}\right)$

$y^{*}=Y(x, y, \in)=y+\in \eta(x, y)+O\left(\in^{2}\right)$

Where $O\left(\in^{2}\right)$ is acting the Tayler expansion about $\in=0$ at the second derivatives

If and only if $X^{(n)} G=0$ when $G=0$ where $X^{(n)}$ is the $\mathrm{n}^{\text {th }}$ extension of the infinitesimal generator X introduced in the following:

$X=\zeta(x, y) \frac{\partial}{\partial x}+\eta(x, y) \frac{\partial}{\partial y}$

Then $\mathrm{G}$ is some function of the invariants write as:

$u(x, y), v_{1}\left(x, y, y^{\prime}\right), \ldots, v_{n}\left(x, y, y^{\prime}, \ldots, y^{(n)}\right)$

Which implies:

$X u(x, y)=0$

Then 
$X v_{j}\left(x, y, y^{\prime}, \ldots, y^{(j)}\right)=0$ with $\frac{\partial v_{j}}{\partial y^{(j)}} \neq 0$, for $k=1,2, \ldots, n$

For the $\mathrm{n}^{\text {th }}$ extension of the group (3.2) and (3.3) we have $\mathrm{u}^{*}=\mathrm{u}, v_{j}^{*}=v_{j}$

For $\mathrm{k}=1,2, \ldots, \mathrm{n} ; v_{j}\left(x, y, y^{\prime}, \ldots, y^{(j)}\right)$ is constant of integration of the characteristic equations given as:

$\frac{d x}{\zeta(x, y)}=\frac{d y}{\eta(x, y)}=\frac{d y^{\prime}}{\eta^{(1)}\left(x, y, y^{\prime}\right)}=\ldots=\frac{d y^{(j)}}{\eta^{(j)}\left(x, y, y^{\prime}, \ldots, y^{(j)}\right)}$

Where $\eta^{(j)}$ introduced by :

$\eta^{(j)}\left(x, y, y^{\prime}, \ldots, y^{(j)}\right)=\frac{D \eta^{(j-1)}}{D x}-y^{(j)} \frac{D \zeta(x, y)}{D x}, j=1,2, \ldots$.

For any set of invariants (2.5) the ODE (2.1) turn into:

$Z\left(u, v_{1}, v_{2}, \ldots, v_{n}\right)=0$

For some function $Z\left(u, v_{1}, v_{2}, \ldots, v_{n}\right)$.

\section{Applications for ODEs}

In this part, we give algorithm to established some problems for ODEs how computed reduction order by using symmetries,

\section{1-Algorithm}

Step1: Write the vector field of the form:

$$
X=\zeta(x, y) \frac{\partial}{\partial x}+\eta(x, y) \frac{\partial}{\partial y}
$$

Step2: Find the $\mathrm{n}^{\text {th }}$ prolongation for equation (2.1) in style:

$$
X^{[n]}=X+\eta^{(1)} \frac{\partial}{\partial y^{\prime}}+\ldots+\eta^{(n)} \frac{\partial}{\partial y^{(n)}}
$$

Step3: Applying the prolongation $\mathrm{X}^{[\mathrm{n}]}$ on equation (2.1) found in Step2 as:

$$
\left.X^{[n]}\left(y^{(n)}-g\left(x, y, y^{\prime}, y^{\prime \prime}, \ldots, y^{(n-1)}\right)\right)\right|_{(2.1)=0}=0
$$

Step4: By using expansion of $\zeta^{(1)}, \zeta^{(2)}, \zeta^{(3)}$ from[10] and $\zeta^{(4)}, \zeta^{(5)}, \ldots, \zeta^{(n)}$ found by using maple package.

Step5: Replacing $y^{(n)}$ by $g\left(x, y, y^{\prime}, y^{\prime \prime}, \ldots, y^{(n-1)}\right)$.

Step6: Find the general solution .

Step7: Reduce order of ODE by using new vector field found in Step6.

Step8: Appling the $\mathrm{n}^{\text {th }}$ prolongation for the new vector field .

Step9: Find invariance using $X^{[n]} I=0$ whose characteristic system is :

$\frac{d x}{\zeta(x, y)}=\frac{d y}{\eta(x, y)}=\frac{d y^{\prime}}{\eta^{(1)}\left(x, y, y^{\prime}\right)}=\ldots=\frac{d y^{(j)}}{\eta^{(j)}\left(x, y, y^{\prime}, \ldots, y^{(j)}\right)}$

Step10: Solving system in Step 9 and substituting solution in (3.1) at end we get the general solution.

Example(3.1): reduce the ODE given in the form:

$y^{2} y^{\prime \prime}+x y^{\prime 2}-y \quad y^{\prime}=0$

To solve (3.1) we must write the vector field as:

$$
X=\zeta(x, y) \frac{\partial}{\partial x}+\eta(x, y) \frac{\partial}{\partial y}
$$

We need the 2-prolongation of (3.2) then:

$$
X^{[2]}=X+\eta^{(1)} \frac{\partial}{\partial y^{\prime}}+\eta^{(2)} \frac{\partial}{\partial y^{\prime \prime}}
$$

Applying equation (3.3) in equation (3.1) given as: 


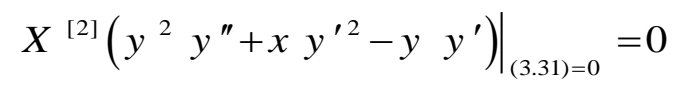

Then

$$
y^{2} \eta^{(2)}+2 x y^{\prime} \eta^{(1)}-y \eta^{(1)}+\left(2 y y^{\prime \prime}-y^{\prime}\right) \eta+y^{\prime 2} \zeta=0
$$

By substituting $\eta^{(1)}$ and $\eta^{(2)}$ from definition:

$$
\begin{aligned}
& \eta_{x x}+\left(2 \eta_{x y}-\zeta_{x x}\right) y^{\prime}+\left(\eta_{y y}-2 \zeta_{x y}\right) y^{\prime 2}-\zeta_{y y} y^{\prime 3} \\
& +\left[\left(\eta_{y}-2 \zeta_{x}-3 \zeta_{y} y^{\prime}\right) y^{\prime \prime}\right] y^{2}+2 x y^{\prime}\left[\eta_{x}+\left(\eta_{y}-\zeta_{x}\right) y^{\prime}-\zeta_{y} y^{\prime 2}\right] \\
& -y\left[\eta_{x}+\left(\eta_{y}-\zeta_{x}\right) y^{\prime}-\zeta_{y} y^{\prime 2}\right]+\eta\left(2 y y^{\prime \prime}-y^{\prime}\right)+y^{\prime 2} \zeta=0
\end{aligned}
$$

Now, substituting $y^{\prime \prime} b y\left(\frac{y y^{\prime}-x y^{\prime 2}}{y^{2}}\right)$ we find:

$$
\begin{aligned}
& \eta_{x x}+\left(2 \eta_{x y}-\zeta_{x x}\right) y^{\prime}+\left(\eta_{y y}-2 \zeta_{x y}\right) y^{\prime 2}-\zeta_{y y} y^{\prime 3}+\left[\left(\eta_{y}-2 \zeta_{x}-3 \zeta_{y} y^{\prime}\right)\left(\frac{y y^{\prime}-x y^{\prime 2}}{y^{2}}\right)\right] y^{2} \\
& +2 x y^{\prime}\left[\eta_{x}+\left(\eta_{y}-\zeta_{x}\right) y^{\prime}-\zeta_{y} y^{\prime 2}\right]-y\left[\eta_{x}+\left(\eta_{y}-\zeta_{x}\right) y^{\prime}-\zeta_{y} y^{\prime 2}\right] \\
& +\eta\left[2 y\left(\frac{y y^{\prime}-x y^{\prime 2}}{y^{2}}\right)-y^{\prime}\right]+y^{\prime 2} \zeta=0
\end{aligned}
$$

By separation of the coefficient $y^{\prime 2}, y^{\prime 3}, y^{\prime}, y y^{\prime 2}, y y^{\prime}, y^{2} y^{\prime 2}$ and $y^{2} y^{\prime}$.

$$
\begin{aligned}
& y^{\prime 2}: \zeta+2 x\left(\eta_{y}-\zeta_{x}\right)-\frac{2 x}{y} \eta-x\left(\eta_{y}-2 \zeta_{x}\right)=0 \\
& y^{\prime 3}:-2 x \zeta_{y}-\zeta_{y y}+3 x \zeta_{y}=0 \\
& y^{\prime}: 2 x \eta_{x}+\eta=0 \\
& y y^{\prime 2}:-4 \zeta_{y}=0 \\
& y y^{\prime}:-\zeta_{x}-2 \zeta_{y}=0 \\
& y^{2} y^{\prime 2}: \eta_{y y}-2 \zeta_{x y}=0 \\
& y^{2} y^{\prime}: 2 \eta_{x y}-\zeta_{x x}=0 \\
& 1: \eta_{x x} y^{2}-\eta_{x} y=0
\end{aligned}
$$

Then the general solution of above system is :

$$
\zeta(x, y)=c_{1} x
$$

$\eta(x, y)=c_{1} y$

Now , to reduce order of ODE write the vector field as follows:

$X_{1}=x \frac{\partial}{\partial x}+y \frac{\partial}{\partial y}$

The $2^{\text {nd }}$ prolongation of $\mathrm{X}_{1}$ is :

$X_{1}^{[2]}=X_{1}+y \frac{\partial}{\partial y}+y^{\prime} \frac{\partial}{\partial y^{\prime}}+y^{\prime \prime} \frac{\partial}{\partial y^{\prime \prime}}$

Then

$$
X_{1}^{[2]}=X_{1}+y \frac{\partial}{\partial y}+0 \frac{\partial}{\partial y^{\prime}}+y^{\prime \prime} \frac{\partial}{\partial y^{\prime \prime}}
$$

We find invariance using $X^{[2]} I=0$ whose characteristic system is :

$\frac{d x}{x}=\frac{d y}{y}=\frac{d y^{\prime}}{0}=\frac{d y^{\prime \prime}}{-y^{\prime \prime}}$

Solving equation (3.21) we result two invariance of $\mathrm{X}^{[2]}$ given by:

$$
\begin{gathered}
\frac{y}{x}=u \\
v=y^{\prime}
\end{gathered}
$$


To write the given ODE in terms of $\mathrm{u}$ and $\mathrm{v}$ we get:

$y^{2} y^{\prime \prime}=y^{2} \frac{d y^{\prime}}{d x}=y^{2} \frac{d v}{d x}=y^{2} \frac{d v}{d u} \frac{d u}{d x}=u \frac{d v}{d u}(x v-y)$

Using equation ((3.22)-(3.24)) in the ODE of (1) gives:

$u(x v-y) \frac{d v}{d u}+x v^{2}-y v=0$

Dividing equation (3.25) by $\mathrm{x}$ implies that :

$u(v-u) \frac{d v}{d u}+v^{2}-u v=0$

Now, solve equation (3.26) if:

A- $v \neq \boldsymbol{u}$ we get the following:

$$
\frac{d v}{d u}=\frac{-v}{u}
$$

Solve equation (3.27) we find the result:

$v=\frac{c}{u} \rightarrow y^{\prime}=\frac{d y}{d x}=\frac{c}{\frac{y}{x}} \rightarrow \frac{d y}{d x}=\frac{c x}{y}$

Then by solving equation (3.28) we get:

$y= \pm \sqrt{c_{1} x^{2}+c_{2}}$

B- $v=u$

$y^{\prime}=\frac{y}{x} \rightarrow \frac{d y}{d x}=\frac{y}{x}$

Then from equation (3.30) we obtain:

$y=c x$

Example(3.2): reduce the ODE write in style as:

$x^{2} y \frac{d^{2} y}{d x^{2}}+x\left(\frac{d y}{d x}\right)^{2}+y\left(\frac{d y}{d x}\right)-x y^{2}=0$

First write the vector field given as:

$X=\zeta(x, y) \frac{\partial}{\partial x}+\eta(x, y) \frac{\partial}{\partial y}$

To compute equation (3.32) we need the $2^{\text {nd }}$ prolongation given by the following:

$X^{[2]}=X+\eta^{(1)} \frac{\partial}{\partial y^{\prime}}+\eta^{(2)} \frac{\partial}{\partial y^{\prime \prime}}$

Now, applying the formula of equation (3.35) in equation (3.32) we obtain:

$\left.X^{[2]}\left(x^{2} y y^{\prime \prime}+x y^{\prime 2}+y y^{\prime}-x y\right)\right|_{(3.32)=0}=0$

Then

$x^{2} y \eta^{(2)}+2 x y^{\prime} \eta^{(1)}+y \eta^{(1)}+2 x y y^{\prime \prime} \zeta+x^{2} y^{\prime \prime} \eta+y^{\prime 2} \zeta+y^{\prime} \eta-y^{2} \zeta-2 x y \eta=0$

By substituting $\eta^{(1)}$ and $\eta^{(2)}$ from definition are:

$X^{2} y\left[\eta_{x x}+\left(2 \eta_{x y}-\zeta_{x x}\right) y^{\prime}+\left(\eta_{y y}-2 \zeta_{x y}\right) y^{\prime 2}-\zeta_{y y} y^{\prime 3}\right]$

$+\left[\left(\eta_{y}-2 \zeta_{x}-3 \zeta_{y} y^{\prime}\right) y^{\prime \prime}\right]+2 x y^{\prime}\left[\eta_{x}+\left(\eta_{y}-\zeta_{x}\right) y^{\prime}-\zeta_{y} y^{\prime 2}\right]$

$+y\left[\eta_{x}+\left(\eta_{y}-\zeta_{x}\right) y^{\prime}-\zeta_{y} y^{\prime 2}\right]+\left(2 x y \zeta+x^{2} \eta\right) y^{\prime \prime}+y^{\prime 2} \zeta+y^{\prime} \eta$

$-\zeta y^{2}-2 x$ y $\eta=0$

After that, substituting $y^{\prime \prime}$ by $\left(x y^{2}-x y^{\prime 2}-y y^{\prime}\right) \frac{1}{x^{2} y}$ we get: 


$$
\begin{aligned}
& X^{2} y\left[\eta_{x x}+\left(2 \eta_{x y}-\zeta_{x x}\right) y^{\prime}+\left(\eta_{y y}-2 \zeta_{x y}\right) y^{\prime 2}-\zeta_{y y} y^{\prime 3}\right] \\
& +\left[\left(\eta_{y}-2 \zeta_{x}-3 \zeta_{y} y^{\prime}\right)\left(x y^{2}-x y^{\prime 2}-y y^{\prime}\right) \frac{1}{x^{2} y}\right]+2 x y^{\prime}\left[\eta_{x}+\left(\eta_{y}-\zeta_{x}\right) y^{\prime}-\zeta_{y} y^{\prime 2}\right] \\
& +y\left[\eta_{x}+\left(\eta_{y}-\zeta_{x}\right) y^{\prime}-\zeta_{y} y^{\prime 2}\right]+\left(2 x y \zeta+x^{2} \eta\right)\left(x y^{2}-x y^{\prime 2}-y y^{\prime}\right) \frac{1}{x^{2} y}+y^{\prime 2} \zeta+y^{\prime} \eta \\
& -\zeta y^{2}-2 x y \eta=0 \\
& \text { Then } \\
& y^{\prime 2}\left[2 x\left(\eta_{y}-\zeta_{x}\right)-\zeta-x\left(\eta_{y}-2 \zeta_{x}\right)-\frac{x}{y} \eta\right]+y^{\prime}\left[2 x \eta_{x}-\frac{2 y}{x} \zeta\right]+y^{\prime 3}\left[x \zeta_{y}-\zeta_{y}\right] \\
& +y y^{\prime 2}\left[x^{2}\left(\eta_{y y}-2 \zeta_{x y}\right)+3 y \zeta_{y}\right]+y y^{\prime}\left[x^{2}\left(2 \eta_{x y}-\zeta_{x x}\right)+\zeta_{x}\right]+y\left[x^{2} \eta_{x x}-2 x \eta+\eta_{x}\right] \\
& -\zeta y^{2}+x y^{2}\left(\eta_{y}-2 \zeta_{x}\right)+x \eta_{y}+2 y^{2} \zeta-3 x y^{2} y^{\prime} \zeta_{y}=0
\end{aligned}
$$

By separation of the coefficient of the above system:

$$
\begin{aligned}
& y^{\prime 2}: 2 x\left(\eta_{y}-\zeta_{x}\right)-\zeta-x\left(\eta_{y}-2 \zeta_{x}\right)-\frac{x}{y} \eta=0 \\
& y^{\prime}: 2 x \eta_{x}-\frac{2 y}{x} \zeta=0 \\
& y^{\prime 3}: x \zeta_{y}-\zeta_{y}=0 \\
& y y^{\prime 2}: x^{2}\left(\eta_{y y}-2 \zeta_{x y}\right)+3 y \zeta_{y}=0 \\
& y y^{\prime}: x^{2}\left(2 \eta_{x y}-\zeta_{x x}\right)=0 \\
& y^{2} y^{\prime}:-3 x \zeta_{y}=0 \\
& 1: y\left(x^{2} \eta_{x x}-2 x \eta+\eta_{x}\right)-\zeta y^{2}+x y^{2}\left(\eta_{y}-2 \zeta_{x}\right)+x \eta_{y}+2 y^{2} \zeta=0
\end{aligned}
$$

Then the general solution of above system are:

$$
\begin{aligned}
& \zeta(x, y)=0 \\
& \eta(x, y)=c_{1} y
\end{aligned}
$$

The generator given as :

$$
X_{1}=y \frac{\partial}{\partial y}
$$

The $2^{\text {nd }}$ prolongation of $\mathrm{X}_{1}$ is :

$X_{1}^{[2]}=X_{1}+y \frac{\partial}{\partial y}+y^{\prime} \frac{\partial}{\partial y^{\prime}}+y^{\prime \prime} \frac{\partial}{\partial y^{\prime \prime}}$

Then

$X_{1}^{[2]}=0 \frac{\partial}{\partial x}+y \frac{\partial}{\partial y}+y^{\prime} \frac{\partial}{\partial y^{\prime}}+y^{\prime \prime} \frac{\partial}{\partial y^{\prime \prime}}$

Solving the characteristic system $X^{[2]} \boldsymbol{I}=\mathbf{O}$ is given the differential invariants .

$$
\begin{aligned}
& \frac{d x}{0}=\frac{d y}{y}=\frac{d y^{\prime}}{y^{\prime}}=\frac{d y^{\prime \prime}}{y^{\prime \prime}} \\
& \text { Then } \\
& u=x \\
& v=\frac{y^{\prime}}{y} \rightarrow y^{\prime}=v \quad y \\
& y^{\prime \prime}=y \frac{d v}{d u}+v^{2} y
\end{aligned}
$$

Now, substituting equation (3.55) in equation (3.32) we obtain: 
$x^{2} y^{2}\left[\frac{d v}{d u}+v^{2}\right]+x y^{2} v^{2}+y^{2} v-x y^{2}=0$

Dividing equation (3.56) on $x^{2} y^{2}$ we get :

$\left[\frac{d v}{d u}+v^{2}\right]+\frac{v^{2}}{u}+\frac{v}{u^{2}}-\frac{1}{u}=0$

Multiplying equation (3.57) by $\mathrm{u}^{2}$ we find:

$u^{2}\left[\frac{d v}{d u}+v^{2}\right]+v^{2} u+v-u=0$

Then

$\frac{d v}{d u}=\frac{1}{u}-\frac{v^{2}}{u}-\frac{v}{u}-v^{2}$

Then the equation (3.59) reduced $1^{\text {st }}$ order ODE .

\section{Reduction of Order for PDEs}

In this part, established reduction order for PDEs the technique is basically the same as for ODEs Now, consider the second order of PDE is :

$G\left(t, x, u, u_{x}, u_{t}, u_{x x}, u_{x t}, u_{t t}\right)=u_{t}-g\left(t, x, u, u_{x}, u_{t}, u_{x x}, u_{x t}, u_{t t}\right)=0$

The point symmetries of the form:

$t^{*}=T(t, x, u ; \in)=t+\in \tau(t, x, u)+O\left(\in^{2}\right)$

$x^{*}=X(t, x, u ; \in)=x+\in \zeta(t, x, u)+O\left(\in^{2}\right)$

$u^{*}=U(t, x, u ; \in)=u+\in \eta(t, x, u)+O\left(\in^{2}\right)$

If and only if $\boldsymbol{X}^{{ }^{(2)} \boldsymbol{G}}=\mathrm{O}$ when $\boldsymbol{G}=\mathrm{O}$ where $X^{(2)}$ is the $2^{\text {th }}$ extension of the infinitesimal generator $\mathrm{X}$ introduced in the following:

$X=\zeta(t, x, u) \frac{\partial}{\partial x}+\tau(t, x, u) \frac{\partial}{\partial t}+\eta(t, x, u) \frac{\partial}{\partial u}$

Then $\mathrm{G}$ is some function of the invariants write as:

$I(t, x), I_{1}(t, x, u), \ldots, I_{2}\left(t, x, u, u_{x}, u_{t}, u_{x x}, u_{x t}, u_{t t}\right)$

Which implies that:

$X I=0$

This condition (4.5) can be expressed by using the characteristic of the group which is:

$Q=\eta-\zeta u_{x}-\tau u_{t}$

From condition $(4.5)$, the surface $\mathrm{u}=\mathrm{u}(\mathrm{t}, \mathrm{x})$ is invariant provided that:

$Q=\mathrm{O}$ when $\mathrm{u}=\mathrm{u}(\mathrm{t}, \mathrm{x})$

In other words from [7], every invariant solution satisfies the invariant surface condition write as:

$Q \equiv \eta-\zeta u_{x}-\tau u_{t}=0$

Now, suppose that $\zeta$ and $\boldsymbol{\eta}$ are not both zero then the invariant surface condition is a 1-order quasilinear PDE can be solved by the method of characteristic equation are:

$\frac{d x}{\zeta}=\frac{d t}{\tau}=\frac{d u}{\eta}$

\section{Applications for PDEs}

In the following, we confirmed algorithm for reducing order of PDE by using symmetry .Moreover calculated some examples about it.

\section{2-Algorithm}

Step1: Write the vector field of the form:

$X=\zeta(t, x, u) \frac{\partial}{\partial x}+\tau(t, x, u) \frac{\partial}{\partial t}+\eta(t, x, u) \frac{\partial}{\partial u}$

Step2: Find the $2^{\text {th }}$ prolongation for equation (4.1) in style:

$X^{[2]}=X+\zeta^{t} \frac{\partial}{\partial u_{t}}+\zeta^{x} \frac{\partial}{\partial u_{x}}+\zeta^{t x} \frac{\partial}{\partial u_{t x}}+\zeta^{t t} \frac{\partial}{\partial u_{t t}}+\zeta^{x x} \frac{\partial}{\partial u_{x x}}$

Step3: Applying the prolongation $\mathrm{X}^{[2]}$ on equation (4.1) found in Step2 as: 
$\left.X^{[2]}\left(u_{t}-g\left(t, x, u, u_{x}, u_{t}, u_{x x}, u_{x t}, u_{t t}\right)\right)\right|_{(4.1)=0}=0$

Step4: By using expansion $\zeta^{t}, \zeta^{x}, \zeta^{t x}, \zeta^{x x}$ and $\zeta^{t t}$ from[11]

Step5: Replacing $u_{t}$ by $g\left(t, x, u, u_{x}, u_{t}, u_{x x}, u_{x t}, u_{t t}\right)$.

Step6: Find the general solution .

Step7: Reduce order of PDE by using new vector field found in Step6.

Step8: Appling the $2^{\text {th }}$ prolongation for the new vector field .

Step9: By solving the invariance $Q \equiv \eta-\zeta u_{x}-\tau u_{t}=0$ whose characteristic equation:

$\frac{d x}{\zeta}=\frac{d t}{\tau}=\frac{d u}{\eta}$

Step10: Solving the characteristic in Step9 and substituting solution in (1) at end we get the general solution.

Example(5.1): reduce the PDE given in the form:

$$
\frac{\partial u}{\partial t}=\frac{\partial^{2} u}{\partial x^{2}}+\frac{\partial u}{\partial x}
$$

First write the vector field given as:

$$
X=\zeta(t, x, u) \frac{\partial}{\partial x}+\tau(t, x, u) \frac{\partial}{\partial t}+\eta(t, x, u) \frac{\partial}{\partial u}
$$

The vector field in equation (5.2) we need the $2^{\text {nd }}$ prolongation given by:

$$
X^{[2]}=X+\zeta^{t} \frac{\partial}{\partial u_{t}}+\zeta^{x} \frac{\partial}{\partial u_{x}}+\zeta^{t x} \frac{\partial}{\partial u_{t x}}+\zeta^{t t} \frac{\partial}{\partial u_{t t}}+\zeta^{x x} \frac{\partial}{\partial u_{x x}}
$$

Now, applying formula of equation (5.3) in equation (5.1) we get :

$$
\left.X^{[2]}\left(u_{t}-u_{x x}-u_{x}\right)\right|_{(1)=0}=0
$$

Then

$$
\zeta^{t}-\zeta^{x x}-\zeta^{x}=0
$$

By using definitions of $\zeta^{t}, \zeta^{x}$ and $\zeta^{x x}$ we obtain the following:

$\eta_{t}+u_{t}\left(\eta_{u}-\tau_{t}\right)-u_{t}^{2} \tau_{u}-u_{x} \zeta_{t}-u_{x} u_{t} \zeta_{u}-\eta_{x x}-2 u_{x} \eta_{x u}-u_{x x} \eta_{u}-u_{x}^{2} \eta_{u u}+2 u_{x x} \zeta_{x}+u_{x} \zeta_{x x}$

$+u_{x}^{2} \zeta_{x u}+\tau_{u}\left(u_{t} u_{x x}+2 u_{x} u_{x t}\right)+u_{x}^{3} \zeta_{u u}+2 u_{x t} \tau_{x}+u_{t} \tau_{x x}+2 u_{x} u_{t} \tau_{x u}+u_{x}^{2} u_{t} \tau_{u u}+3 u_{x} u_{x x} \zeta_{u}-\eta_{x}$

$-u_{x}\left(\eta_{u}-\zeta_{x}\right)+u_{x}^{2} \zeta_{u}+u_{t} \tau_{x}+u_{x} u_{t} \tau_{u}=0$

Now , replacing $u_{t}$ by $u_{x x}+u_{x}$ we result:

$$
\begin{aligned}
& \eta_{t}+\left(u_{x x}+u_{x}\right)\left(\eta_{u}-\tau_{t}\right)-\left(u_{x x}+u_{x}\right)^{2} \tau_{u}-u_{x} \zeta_{t}-\left(u_{x x}+u_{x}\right) u_{x} \zeta_{u}-\eta_{x x}-2 u_{x} \eta_{x u} \\
& -u_{x x} \eta_{u}-u_{x}^{2} \eta_{u u}+2 u_{x x} \zeta_{x}+u_{x} \zeta_{x x}+u_{x}^{2} \zeta_{x u}+\tau_{u}\left(\left(u_{x x}+u_{x}\right) u_{x x}+2 u_{x} u_{x t}\right) \\
& +u_{x}^{3} \zeta_{u u}+2 u_{x t} \tau_{x}+\left(u_{x x}+u_{x}\right) \tau_{x x}+2\left(u_{x x}+u_{x}\right) u_{x} \tau_{x u}+\left(u_{x x}+u_{x}\right) u_{x}^{2} \tau_{u u} \\
& +3 u_{x} u_{x x} \zeta_{u}-\eta_{x}-u_{x}\left(\eta_{u}-\zeta_{x}\right)+u_{x}^{2} \zeta_{u}+\left(u_{x x}+u_{x}\right) \tau_{x}+\left(u_{x x}+u_{x}\right) u_{x} \tau_{u}=0 \\
& u_{x x}\left(\tau_{x x}+\tau_{x}-\tau_{t}+2 \zeta_{x}\right)+u_{x}\left(\zeta_{x x}+\zeta_{x}+\tau_{x x}+\tau_{x}-\tau_{t}-\zeta_{t}-2 \eta_{x u}\right) \\
& +u_{x}^{2}\left(\zeta_{x u}+2 \tau_{x u}-\eta_{u u}\right)+u_{x} u_{x x}\left(2 \tau_{x u}+2 \zeta_{u}\right)+u_{x}^{3}\left(\zeta_{u u}+\tau_{u u}\right) \\
& +u_{x}^{2} u_{x x} \tau_{u u}+2 u_{x} u_{x t} \tau_{u}+2 u_{x t} \tau_{x}-\eta_{x x}-\eta_{x}+\eta_{t}=0
\end{aligned}
$$

By using separation of the coefficient :

$$
\begin{aligned}
& u_{x x}: \tau_{x x}+\tau_{x}-\tau_{t}+2 \zeta_{x}=0 \\
& u_{x}: \zeta_{x x}+\zeta_{x}+\tau_{x x}+\tau_{x}-\tau_{t}-\zeta_{t}-2 \eta_{x u}=0 \\
& u_{x}^{2}: \zeta_{x u}+2 \tau_{x u}-\eta_{u u}=0
\end{aligned}
$$




$$
\begin{aligned}
& u_{x} u_{x x}: 2 \tau_{x u}+2 \zeta_{u}=0 \\
& u_{x}^{3}: \zeta_{u u}+\tau_{u u}=0 \\
& u_{x}^{2} u_{x x}: \tau_{u u}=0 \\
& u_{x} u_{x t}: 2 \tau_{u}=0 \\
& u_{x t}: 2 \tau_{x}=0
\end{aligned}
$$

Then the general solution of above system is :

$$
\begin{aligned}
& \zeta(t, x, u)=\frac{1}{2}\left(c_{1} x-c_{2}-4 c_{4}\right) t+\frac{1}{2} x c_{2}+c_{6} \\
& \tau(t, x, u)=\frac{1}{2} c_{1} t^{2}+c_{2} t+c_{3} \\
& \eta(t, x, u)=-\frac{1}{8}\left[\left(t^{2}+(2 x+2) t+x^{2}\right) c_{1}-8 c_{4}(t+x)-8 c_{5}\right] u+\alpha(t, x)
\end{aligned}
$$

Then the Lie symmetries given as:

$$
\begin{aligned}
& X_{1}=\frac{1}{2} x t \frac{\partial}{\partial x}+\frac{1}{2} t^{2} \frac{\partial}{\partial t}-\frac{1}{8}\left(t^{2}+(2 x+2) t+x^{2}\right) u \frac{\partial}{\partial u} \\
& X_{2}=\left(-t+\frac{1}{2} x\right) \frac{\partial}{\partial x}+t \frac{\partial}{\partial t} \\
& X_{3}=\frac{\partial}{\partial t} \\
& X_{4}=-4 t \frac{\partial}{\partial x}-(t+x) u \frac{\partial}{\partial u} \\
& X_{5}=u \frac{\partial}{\partial u} \\
& X_{6}=\frac{\partial}{\partial x} \\
& X_{\alpha}=\alpha(t, x) \frac{\partial}{\partial u}
\end{aligned}
$$

Using the symmetries as:

$X=X_{1}=\frac{1}{2} x t \frac{\partial}{\partial x}+\frac{1}{2} t^{2} \frac{\partial}{\partial t}-\frac{1}{8}\left(t^{2}+(2 x+2) t+x^{2}\right) u \frac{\partial}{\partial u}$

Then

$$
\frac{d x}{\frac{1}{2} x t}=\frac{d t}{\frac{1}{2} t^{2}}=\frac{d u}{-\frac{1}{8}\left(t^{2}+(2 x+2) t+x^{2}\right) u}
$$

By solving equation (5.28) we find the following :

$$
\zeta(t, x)=\frac{x}{t}
$$

$v(t, x)=\varphi(\zeta) t^{(2 x+2)} e^{-\frac{1}{4}\left(t-\frac{x^{2}}{t}\right)}$

By using (5.29) and (5.30), we find $\frac{\partial u}{\partial t}, \frac{\partial u}{\partial x}$ and $\frac{\partial^{2} u}{\partial x^{2}}$ we get:

$$
\begin{aligned}
& \frac{\partial u}{\partial t}=\frac{\partial v}{\partial \zeta} \cdot \frac{\partial \zeta}{\partial t}=\left[\frac{x t^{(2 x+2)}}{t^{2}} \frac{\partial \varphi}{\partial \zeta}+\varphi\left((2 x+2) t^{2 x+1}-\frac{t^{(2 x+2)}}{4}\left(1+\frac{x^{2}}{t^{2}}\right)\right)\right] e^{-\frac{1}{4}\left(t-\frac{x^{2}}{t^{2}}\right)} \\
& \frac{\partial u}{\partial x}=\frac{\partial v}{\partial \zeta} \cdot \frac{\partial \zeta}{\partial x}=\left[\frac{t^{(2 x+2)}}{t} \frac{\partial \varphi}{\partial \zeta}+\varphi\left(2 t^{2 x+1}+\frac{x}{2 t}\right)\right] e^{-\frac{1}{4}\left(t-\frac{x^{2}}{t^{2}}\right)}
\end{aligned}
$$


$\frac{\partial^{2} u}{\partial x^{2}}=\left[\frac{\partial^{2} \varphi}{\partial \zeta^{2}} \frac{t^{2 x+2}}{t^{2}}+\frac{\partial \varphi}{\partial \zeta}\left(\frac{x t^{2 x+2}}{2 t^{2}}+\frac{4 t^{2 x+2}}{t}+\frac{x}{2 t^{2}}\right)+\varphi\left(\frac{x}{2 t}\left(2 t^{2 x+2}+\frac{x}{2 t}\right)+4 t^{2 x+2}+\frac{1}{2 t}\right)\right] e^{-\frac{1}{4}\left(t-\frac{x^{2}}{t}\right)}$

Now , substituting equations ((5.31)-(5.33)) in equation (5.1) we obtain the ODE :

$\varphi_{\zeta \zeta}-2 \zeta \varphi_{\zeta}+\zeta^{2} \varphi=0$

By solving equation (5.34) we find:

$\varphi(\zeta)=c_{1} e^{\frac{1}{2} \zeta^{2}} \cos (\zeta)+c_{2} e^{\frac{1}{2} \zeta^{2}} \sin (\zeta)$

Then

$u(t, x)=c_{1} e^{\frac{1}{2} \frac{x^{2}}{t^{2}}} \cos \left(\frac{x}{t}\right)+c_{2} e^{\frac{1}{2} \frac{x^{2}}{t^{2}}} \sin \left(\frac{x}{t}\right)$

\section{Discussion and Conclusion}

Lie group methods is finest for all techniques yet based on algorithm established above .In this work , introduced 2-algorithms for ODEs and PDEs, the first algorithm is applied to reduce the order of ODEs and the second algorithm is applied to reduce PDEsto ODEs . Also give some applications for ODEs and PDEs to obtain the exact solutions.

\section{Acknowledgement}

The authors thank of Mustansiriyah University / College of science / Department of Mathematics and Your esteemed journal for their supported this work .

\section{CONFLICT OF INTERESTS}

There are no conflicts of interest.

\section{References}

[1] G.W. Bluman and S. Kumei, "Symmetries and Differential Equations", Appl. Math. Sci. 81, Springer-Verlag, Berlin, 1989.

[2] P.J. Olver ,"Applications of Lie Groups to Differential Equations", Springer-Verlag, New York, 1986.

[3] L.V. Ovsiannikov, "Group Analysis of Differential Equations", Academic Press, New York, 1982.

[4] P. J. Olver, "Applications of Lie Groups to Differential Equations", Springer-Verlag, New York, 1993.

[5] P. A. Clarkson and E. L. Mansfield ,"On a Shallow Water Wave Equation, Nonlinearity 7 Ž", 9751000, (1994).

[6] P. A. Clarkson, "Non-classical Symmetry Reductions of the Boussinesq Equation", Chaos, Solitons and Fractals $5 \check{Z}, 22612301,1995$.

[7] P.E. Hydon, "Symmetry Method for Differential Equations: Abe-ginner's Guide", Cambridge University Press, Cambridge, 2000.

[8] Lie and F. Engel, "Theorie der Transformationsgruppen, Teubner, Leipzig", Germany, 1890.

[9] G.W. Bluman, and J.D. Cole, "The general similarity solution of the heat equation", J. Math, Mech, pp. 10251042,1969 .

[10] N.H. Ibragimov," CRC Handbook of Lie Group Analysis of Differential Equation", Vol. I, CRC Press, USA, 1994.

[11] Y.N. Grigoriev , N.H. Ibragimov, V.F. Kovalev and S.V. Meleshko, " Symmetries of Integro-Differential Equations", With Applications in Mechanics and Plasma Physics, Lect. Notes phys.806 (Springer Dordrecht), DOI 10.1007/978-90-481-3797-8, 2010.

\section{تخفيض المعادلات التفاضلية باستخدام زمرلي}

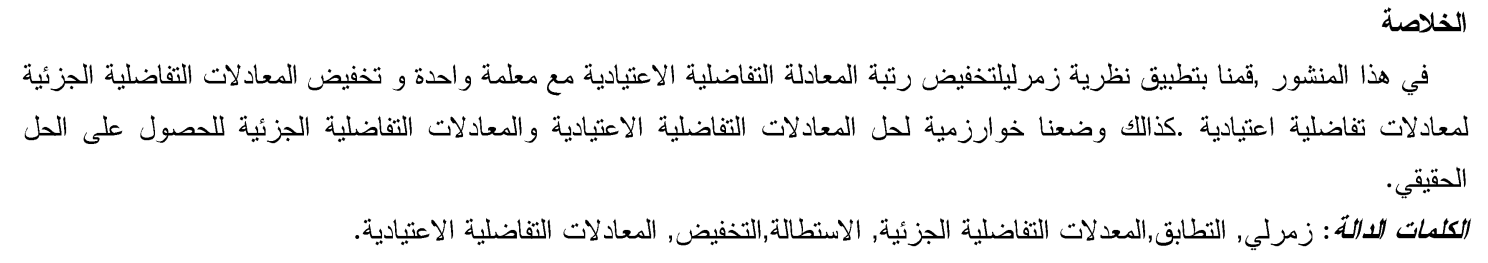

